\title{
Review: stage based interventions did not influence smoking behaviour
}

Riemsma RP, Pattenden J, Bridle C, et al. Systematic review of the effectiveness of stage based interventions to promote smoking cessation. BMJ 2003;326:1175-7.

\section{Q Do stage based interventions change smoking behaviour in people who smoke?}

\section{METHODS}

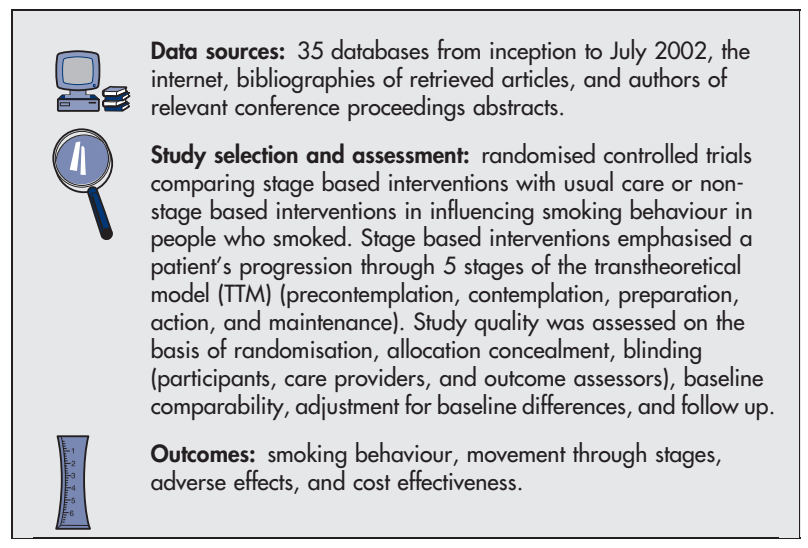

\section{MAIN RESULTS}

23 studies met the selection criteria. Study quality was mixed, ranging from 2 to 12 out of 13 for the number of quality criteria present. Information on validation of the instrument for assessing stage of change was present in 2 trials. 8 trials showed an increase in smoking cessation with a stage based intervention compared with usual care ( 7 trials) or a non-stage based intervention ( 1 trial). 12 trials showed no difference ( 5 compared with usual care and 8 compared with a non-stage based intervention). 3 trials were inconclusive because multiple outcomes were used for smoking behaviour, $>1$ stage based intervention was evaluated, or the direction of effect differed at different follow up time points. In 10 trials that reported movement through stages, 1 trial showed statistically significant effects in favour of a stage based intervention.

For correspondence: Mr R P Riemsma, Centre for Reviews and Dissemination, University of York, York, UK. rpr1@york.ac.uk

Source of funding: NHS Research and Development Health Technology Assessment Programme.

\section{CONCLUSION}

Stage based interventions were no more effective than non-stage based interventions or usual care in changing smoking behaviour in people who smoke.

\section{Commentary}

The health benefits of smoking cessation are well documented although quitting smoking can be very difficult. Interventions to aid smoking cessation include pharmacological agents such as nicotine replacement therapy ${ }^{1}$ and behavioural approaches, mostly stage based interventions informed by the TM.

The systematic review by Riemsma et al used rigorous methodology to assess the effectiveness of stage based behavioural interventions. Comprehensive, extensive searches were undertaken, and the methodological quality of included studies was independently assessed and reported fully. Details on volume of abstracts obtained and rationale for exclusion of studies, some of which were included in a previous narrative review of $\Pi M$, were not provided. ${ }^{2}$ Heterogeneity of interventions prevented meta-analysis.

Although the outcomes of the review were behaviour change and movement across stages, less than half of the included studies evaluated stage change. Furthermore, problems existed with validity of assessment of stage change. The review has been criticised for lack of clarity regarding whether interventions were stage matched, the descriptive rather than quantitative summary, and the lack of weighting by study quality. ${ }^{3}$ However, most of these issues were discussed in the review by the authors themselves. There is a clear need for high quality trials of stage based behavioural interventions before the TM approach to smoking cessation is abandoned. In the meantime, health professionals should not be discouraged from promoting smoking cessation at every opportunity.

\section{Julie Bruce, RGN, MSc} University of Aberdeen Aberdeen, UK

1 Woolacott NF, Jones L, Forbes CA, et al. The clinical effectiveness and costeffectiveness of bupropion and nicotine replacement therapy for smoking cessation: a systematic review and economic evaluation. Health Technol Assess 2002;6:1-245.

2 Spencer L, Pagell F, Hallion ME, et al. Applying the transtheoretical model to tobacco cessation and prevention: a review of literature. Am J Health Promot 2002;17:7-71.

3 Rapid responses and eletters to Riemsma on BMJ website. http:// bmi.bmijournals.com/cgi/eletters/326/7400/1175 